\title{
Preferencias de niños y niñas en relación con los artrópodos epigeos (Metazoa: Arthropoda) del desierto florido de Chile
}

\author{
Boys' and girls' preference in relation to the epigean arthropods \\ (Metazoa: Arthropoda) of the Chilean flowering desert \\ Jaime Pizarro-Araya ${ }^{1 *}$, Francisco López-Cortés ${ }^{2}$, \\ Stephen M. Jewell ${ }^{3}$, Mary-Carmen Inostroza ${ }^{2}$
}

\section{RESUMEN}

Aunque los problemas medioambientales, incluida la extinción, son de gran importancia en la actualidad, existe un desconocimiento general acerca de la conservación de la biota, en especial de los animales, y en particular de los artrópodos. El interés en los artrópodos empieza durante la niñez, pero investigaciones sobre los conocimientos y preferencias de los niños y niñas chilenos acerca de ellos, a la fecha, no existen. La presente investigación documenta las preferencias taxonómicas y conocimiento morfológico entre género y edad de un grupo de niños y niñas de Educación General Básica en Chile, respecto de los artrópodos epigeos del desierto florido chileno. El presente estudio pone de manifiesto que existen diferencias significativas entre las preferencias asociadas al género de niños y niñas, y que la edad no es un factor determinante frente a tales preferencias. Las preferencias taxonómicas de los niños y niñas en relación con los artrópodos epigeos del desierto florido chileno fueron la mariposa (Insecta: Lepidoptera) (elegida por 33,7\% de los participantes en primera preferencia), seguido por la chinita (Insecta: Coccinelidae) (20,5\%) que fue el más popular en segunda preferencia y la araña (Arachnida: Araneae) (10,5\%) como el más popular en tercera preferencia. Respecto de las diferencias entre las preferencias relacionadas con el género de los escolares, las niñas prefirieron las mariposas (Insecta: Lepidoptera) (30,3\%) y las chinitas (23,2\%) mientras que los niños prefirieron el escorpión (Arachnida: Scorpiones) $(21,4 \%)$, la araña pollito (Arachnida: Theraphosidae) (17,9\%) y la araña (Arachnida: Araneae) (10,4\%). En este trabajo se discute la importancia de estos resultados para la conservación de los artrópodos de estas áreas del norte-centro de Chile.

Palabras clave: percepción infantil, conocimientos, artrópodos, niños, desierto florido.

\begin{abstract}
Even though environmental problems, including the present extinction crisis, are of great importance in today's world, there is a general ignorance regarding the preservation of animals, particularly arthropods. The interest in arthropods begins during childhood, but research on boys' and girls' knowledge and preference in relation to them is non-existent. This research documents the taxonomic preferences and morphological knowledge between age groups and gender of a group of primary-school boys and girls in relation to the epigean arthropods of the Chilean flowering desert. The study shows that significant differences exist in genus preference between boys and girls, and that age is not a determining factor in their preferences. The boys' and girls' taxonomical preferences in relation to the epigean arthropods of Chile's flowering desert were the butterfly (Insecta: Lepidoptera), chosen by $33.7 \%$ of the participants as first option, followed by the ladybug (Insecta: Coccinelidae) (20.5\%), which was the most popular second choice, and spiders (Arachnida: Araneae) (10.5\%), the most popular third choice. In relation to genus preferences, girls preferred butterflies (Insecta: Lepidoptera) (30.3\%) and the ladybug (23.2\%), whereas boys preferred scorpions (Arachnida: Scorpiones) (21.4\%), the tarantula (Arachnida: Theraphosidae) (17.9\%) and spiders (Arachnida: Araneae) (10.4\%). This paper discusses the importance of these results for the preservation of arthropods.
\end{abstract}

Key words: children's perception, previous knowledge, arthropods, children, flowering desert.

\section{Introducción}

Hoy, a pesar del interés en las relaciones entre los seres humanos, el medio ambiente y la importancia del bienestar de tales relaciones, persisten serios problemas ambientales y de conservación (Pimm et al., 1995). Uno de los factores que podrían explicar este fenómeno es

\footnotetext{
1 Laboratorio de Entomología Ecológica, Departamento de Biología, Facultad de Ciencias, Universidad de La Serena, Casilla 599, La Serena, Chile.

2 Departamento de Biología, Facultad de Ciencias, Universidad de La Serena, Casilla 599, La Serena, Chile.

3 Psychology Department, Middlebury College, USA.

* Autor para correspondencia: japizarro@userena.cl
}

Fecha de Recepción: 15 Marzo, 2013.

Fecha de Aceptación: 15 Mayo, 2015. 
el hecho de que las personas se mantienen más aisladas del medio ambiente (Miller, 2005; Tomkins y Tunnicliffe, 2007; Prokop y Tunnicliffe, 2010). Además, la tecnología disponible actualmente (e.g., computadores, Internet, televisión, juegos de video) hace que se destine menos tiempo a explorar o jugar en entornos naturales, lo que disminuye las experiencias personales con la naturaleza y la sensibilidad frente a los problemas ambientales (Miller, 2005). Este alejamiento podría comenzar a temprana edad, y se incrementa con el paso de los años, reconociéndose que los momentos críticos para el conocimiento e integración hacia la naturaleza son la niñez y la adolescencia (Miller, 2005; Prokop y Tunnicliffe, 2010). En este sentido, se ha propuesto que la sensibilidad a la conservación se desarrolla durante la niñez (Howe et al., 1996), y que las experiencias con el medio ambiente durante esta etapa son muy importantes en crear y mejorar las percepciones de los ambientes naturales y de los animales (Bixler et al., 2002). Por ejemplo, asociado a las bajas preferencias en relación con los insectos, los niños y niñas muestran variados errores y falta de conocimientos acerca de ellos (Snaddon y Turner, 2007; Prokop et al., 2011a). Por su parte, Shepardson (1997) mostró que en niños y niñas correspondientes al primer curso de educación escolar básica las interacciones sociales les permiten aprender sobre el proceso de metamorfosis, pero este aprendizaje se restringe a los grupos de insectos más conocidos, con los cuales tienen experiencia directa (e.g., mariposas) (Ramadoss y Poyya-Moli, 2012). Respecto del grado de conocimiento acerca de lo que es un insecto, se ha observado que muchos problemas se originan en la dificultad que los niños tienen en diferenciar a los insectos de otros grupos de artrópodos (i.e., arácnidos, crustáceos) (Shepardson, 2002). Aunque los estudiantes utilizan los caracteres morfológicos de un artrópodo para identificarlo como un insecto, cometen variados errores en elegir cuáles son las características que determinan a un insecto (Shepardson, 2002).

Para las áreas desérticas de Chile se reconoce un alto nivel de endemismo y unicidad taxonómica de la flora, pero un bajo nivel de conocimiento de la artropodofauna (Cepeda-Pizarro et al., 2005). Dichas áreas constituyen zonas de interés para la conservación, debido a que cada cierto número de años están expuestas a aumentos inusualmente altos de las precipitaciones, los que asociados a las oscilaciones de ENOS (El Niño-Oscilación del Sur) (Cepeda-Pizarro et al., 2005) configuran un paisaje en que predomina la vegetación efímera (i.e., geófitas, herbáceas), con un marcado incremento de la cobertura vegetal, y de procesos ecosistémicos locales singulares asociados al aumento de la productividad biológica (Gutiérrez, 2008). Este fenómeno de desarrollo vegetal y floración se denomina "desierto florido", y en términos geográficos ocurre en una extensa zona que se extiende por la franja costera y tierras interiores que se extienden entre los $27-30^{\circ} \mathrm{S}$ (Gutiérrez, 2008). En años secos existe una influencia ocasional de las precipitaciones, estando en la actualidad las áreas del desierto florido expuestas a una intensa presión antrópica, especialmente por el desarrollo inmobiliario y el cambio en el uso del suelo y, en consecuencia, posible perjuicio en su particular biodiversidad.

En esta extensa franja territorial se encuentra inmersa una numerosa población escolar, de la que hasta ahora no existen antecedentes sobre las preferencias que tienen hacia el grupo de los artrópodos epigeos del desierto costero, que corresponde al área natural que circunda las ciudades en que viven, o en la cual se encuentran inmersos sus establecimientos educacionales. En este trabajo se propone que existen diferencias en las preferencias taxonómicas en relación con el género y la edad de los estudiantes y que el conocimiento morfológico que estos tienen de los artrópodos es insuficiente para reconocerlos como grupos distintos, debido a la baja representación que tales contenidos tienen en el currículum escolar. Basados en lo anteriormente documentado, los objetivos del presente trabajo son: i) documentar la preferencia taxonómica que tiene un grupo de niños y niñas de Educación General Básica en relación con los artrópodos epigeos del desierto florido chileno, ii) determinar diferencias entre las preferencias de los artrópodos epigeos, en relación con el género, edad y procedencia de los niños y niñas de este grupo de educación básica y iii) establecer el nivel de conocimiento morfológico de los artrópodos epigeos en distintos grupos de edad.

\section{Materiales y Métodos}

\section{Participantes}

El estudio se realizó en el Laboratorio de Entomología Ecológica del Departamento Biología 
(Universidad de La Serena, La Serena, Chile). La actividad estuvo enmarcada en las XIV (4 y 5 de noviembre del 2008) y XV (6 de octubre del 2009) Semana Nacional de la Ciencia y la Tecnología de EXPLORA-CONICYT en la Universidad de La Serena. Participaron en la presente investigación 183 alumnos (97 niños, 86 niñas) de diez escuelas (nueve urbanas, una rural) de las Regiones de Coquimbo y Atacama, Chile. Los alumnos cursaban desde el Tercero al Octavo Año de Educación General Básica, con edades comprendidas entre los 8 y los 15 años (Promedio = 11,26 años). El procedimiento, el lugar, los materiales y el equipo de investigadores se mantuvieron constantes para todos los participantes.

La herramienta principal de la investigación fue una encuesta (Anexo 1) acerca de las preferencias de los niños y las niñas sobre los artrópodos. Se pidió a cada participante elegir a sus tres "bichitos" -artrópodos epigeos- favoritos del desierto florido, de una lista dada de 24 artrópodos específicos, junto con una línea para señalar otra opción de organismo en caso que el de su preferencia no apareciera mencionado en la lista. Otros datos solicitados en la encuesta incluyeron: nombre, edad, curso, establecimiento educacional y ciudad de residencia del participante (Anexo 1). Se incluyó una hoja en blanco con cada encuesta para que los participantes pudieran hacer un dibujo de su animal favorito. En la Tabla 1 se entregan los antecedentes de las diez escuelas, incluyendo el nombre del establecimiento educacional, la ciudad, la procedencia (i.e., urbana/rural) y la dependencia (i.e., municipal, particular-subvencionado) junto con un desglose de los participantes en relación con el género, cursos de Educación General Básica y edades de los alumnos de cada establecimiento educacional.

\section{Análisis de datos}

Se tabularon todos los datos en una matriz utilizando el software STATISTICA (StatSoft, Inc. 2001), en la que se registraron los datos personales de cada participante (nombre, género, edad, establecimiento y localidad) y sus tres preferencias de artrópodos (usando 1 para la primera, 2 para la segunda y 3 para la tercera preferencia). Se tabuló también información taxonómica (Especie, Clase y Orden) de cada preferencia. Para estimar las preferencias globales de los estudiantes por las especies de artrópodos se analizaron las frecuencias de las tres primeras preferencias utilizando tres pruebas de $\chi^{2}$ de frecuencias (Steel y Torrie, 1989). De la misma manera, se realizaron pruebas de $\chi^{2}$ de independencia para documentar la existencia de diferencias en las preferencias, tanto a escala de Especie, Orden y Clase, relacionadas con el género, la edad y la procedencia de las escuelas (urbana o rural) de los participantes. Para estas pruebas se combinaron las tres preferencias en una sola variable para cada nivel taxonómico (Orden, Clase y Especie), usando el método de crear un conjunto de datos de respuestas múltiples (Thomas y Decady, 2000). Se eligió este proceso ya que permite evaluar las diferencias globales de las preferencias, en vez de analizarlas cada una por separado. Para

Tabla 1. Antecedentes de los establecimientos educacionales de origen de los estudiantes encuestados.

\begin{tabular}{|c|c|c|c|c|c|c|c|c|c|}
\hline \multirow{2}{*}{$\begin{array}{c}\text { Año } \\
\text { encuesta }\end{array}$} & \multirow{2}{*}{$\begin{array}{c}\text { Establecimiento } \\
\text { educacional }\end{array}$} & \multirow{2}{*}{ Ciudad } & \multirow{2}{*}{ Dependencia } & \multirow{2}{*}{ Ubicación } & \multicolumn{3}{|c|}{ Número de estudiantes } & \multirow{2}{*}{$\begin{array}{l}\text { Curso } \\
\text { EGB }\end{array}$} & \multirow{2}{*}{$\begin{array}{c}\text { Edad } \\
\text { (Promedio } \\
\pm \text { DE) }\end{array}$} \\
\hline & & & & & Niños & Niñas & Total & & \\
\hline 2008 & Escuela Básica Arturo Alvear R. & Vallenar & M & Rural & 11 & 15 & 26 & 3,5 y 8 & $9,9 \pm 1,8$ \\
\hline 2008 & Colegio San Antonio & La Serena & P-Sub & Urbano & 8 & 7 & 15 & 4 & $9,5 \pm 0,5$ \\
\hline 2008 & Colegio Básico CEDIN & Coquimbo & P-Sub & Urbano & 11 & 5 & 16 & 7 y 8 & $12,9 \pm 0,8$ \\
\hline 2008 & Colegio Cerro Grande & La Serena & P-Sub & Urbano & 3 & 6 & 9 & 3 & $8,3 \pm 0,5$ \\
\hline 2008 & $\begin{array}{l}\text { Colegio Carlos Condell de } \\
\text { La Haza }\end{array}$ & La Serena & M & Urbano & 8 & 9 & 18 & 6 & $11,7 \pm 0,9$ \\
\hline 2008 & Colegio Javiera Carrera & La Serena & M & Urbano & 0 & 13 & 13 & 8 & $13,6 \pm 0,6$ \\
\hline 2008 & Colegio San Luis & Coquimbo & P-Sub & Urbano & 10 & 4 & 14 & 5 & $10,7 \pm 0,9$ \\
\hline 2009 & Colegio María Educa & La Serena & P-Sub & Urbano & 21 & 1 & 22 & 7 & $12,4 \pm 0,5$ \\
\hline 2009 & Colegio Julia de La Barra & La Serena & P-Sub & Urbano & 10 & 22 & 32 & 6 & $11,4 \pm 0,5$ \\
\hline 2009 & Escuela Diego de Almeyda & Coquimbo & P-Sub & Urbano & 15 & 4 & 19 & 5 & $10,6 \pm 0,5$ \\
\hline
\end{tabular}

M = Municipal; P-Sub = Particular subvencionado; EGB = Educación General Básica.

M: Municipal Public School; P-Sub: State-Funded Private School; EGB: Primary Education. 


\section{ANEXO 1}

\section{Encuesta}

Laboratorio de Entomología Ecológica

Departamento de Biología

Universidad de La Serena

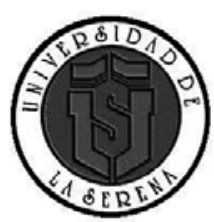

Nombre completo:

Edad: Curso:

Niño: Niña:

Establecimiento educacional: Fecha:

¿Cuál es tu bichito favorito del desierto florido?: Escoge a tus tres favoritos y ponles un número: 1,2 y 3 .

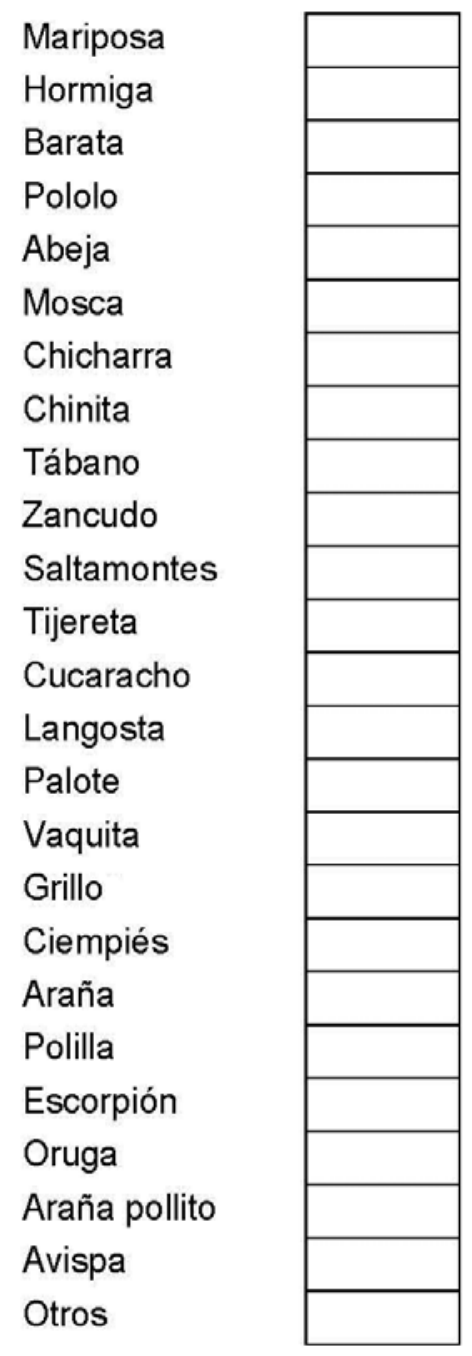

Si tu opción es otros, indícanos cuál es tu bichito: 
documentar el conocimiento de las características morfológicas típicas entre las clases de Arthropoda (i.e., Insecta y Arachnida) se analizaron los 170 dibujos entregados de un total de 183 encuestados, para ello se utilizó una lista de cotejo, la que incluyó tres categorías (i.e., número de patas, número de tagmas -divisiones corporales), cuyas asignaciones eran iguales a 1 punto por carácter. Si en el dibujo se encontraban los tres caracteres morfológicos del artrópodo dibujado (ya sea insecto, arácnido u otro artrópodo), se asignó el valor tres. La ausencia de dichos caracteres en los dibujos, o la asignación incorrecta de caracteres de un tipo de artrópodo a otro, disminuía el puntaje. Se elaboró otra matriz en la que se registró el puntaje del dibujo, y el curso del participante. Se analizaron los datos utilizando un análisis de la varianza (ANOVA) con la variable dependiente del curso (menores a Quinto Año Básico y mayores a Quinto Año Básico) y la variable independiente el puntaje del dibujo. Se utilizó un nivel de incerteza del 5\% $(p \leq 0,05)$ para todas las pruebas estadísticas (Steel y Torrie, 1989).

\section{Resultados}

\section{Preferencia taxonómica de niños y niñas en relación con los artrópodos epigeos}

De los 24 artrópodos señalados en la lista solo a 16 de ellos le fue asignada una primera preferencia, a 22 una segunda preferencia y a 24 la tercera preferencia. Las tres pruebas de $\chi^{2}$ para el análisis de preferencia fueron significativas y dan cuenta que existen artrópodos que son preferidos. Para la prueba de artrópodo favorito $\left(\chi_{(15)}^{2}=358,989, p=0,000\right)$, la mariposa fue la más popular, elegida por $33,7 \%$ de los participantes en primera preferencia. Para la segunda preferencia $\left(\chi_{(21)}^{2}=243,000, p=0,000\right)$, la chinita y el escorpión fueron los artrópodos más elegidos, con $20,5 \%$ y $17,0 \%$ de los participantes, respectivamente. La última prueba, del tercer favorito, fue significativa $\left(\chi_{(23)}^{2}=72,667, p=0,000\right)$, pero fue más equilibrada en general, donde el artrópodo más elegido, la araña, fue la selección de 10,5\% de los participantes (Figura 1).

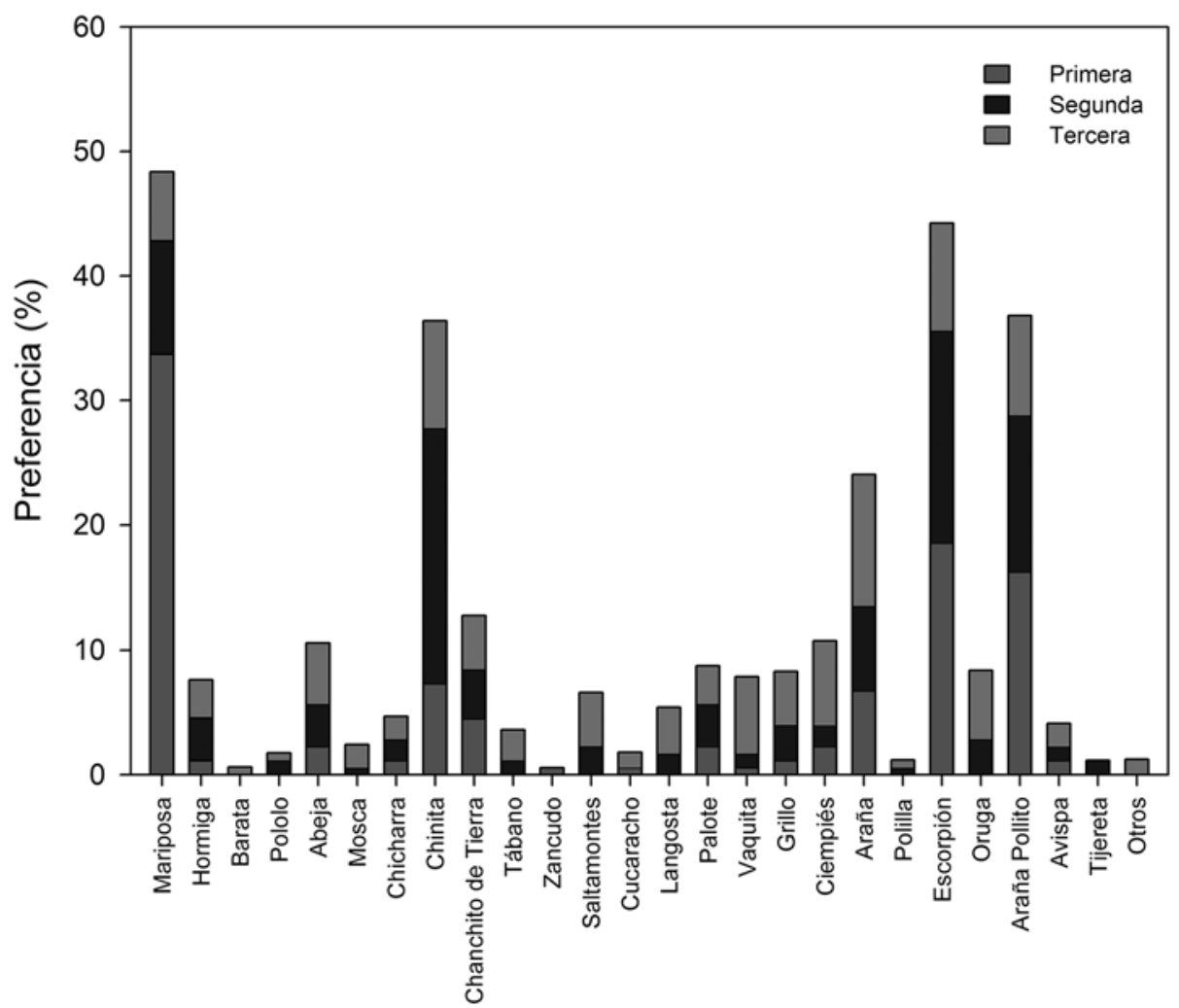

Organismo

Figura 1. Preferencias para los diferentes tipos de artrópodos. 
Para detectar los patrones generales de los resultados se agruparon los datos de las tres preferencias (Figura 1). La mariposa fue lo más popular, elegida por el $46,7 \%$ de los participantes como el artrópodo favorito. Otros artrópodos populares incluyeron el escorpión (Scorpiones) (42,3\%), la araña pollito (Araneae: Theraphosidae) $(35,2 \%)$, la chinita (Coleoptera: Coccinelidae) $(34,6 \%$ ) y la araña (Arachnida: Araneae) (22,5\%) (Figura 1).

\section{Preferencias de los artrópodos epigeos en relación con el género y edad de los niños y niñas}

Los análisis sobre las preferencias de los artrópodos en cada jerarquía taxonómica (i.e., Especie, Orden y Clase) difirieron de manera significativa respecto del género de los participantes, pero no respecto de la edad de los mismos. La prueba entre Género y Especie tuvo un valor de $\chi^{2}{ }_{(26)}=$ 256,$7 ; p=0,000$. Las niñas prefirieron las mariposas y las chinitas mientras que los niños prefirieron el escorpión, la araña y la araña pollito (Figura 2).
Para Género y Orden $\left(\chi_{(13)}^{2}=213,140, p=\right.$ $0,000)$ los niños eligieron los Ordenes Scorpiones y Araneae (Arachnida) y las niñas los Órdenes Lepidoptera y Coleoptera (Insecta) (Figura 3 ). Finalmente, entre Género y Clase $\left(\chi_{(5)}^{2}=97,244\right.$, $p=0,000)$ las niñas prefirieron a los organismos de la Clase Insecta, mientras que los niños prefirieron los organismos de las Clases Arachnida, Chilopoda y ligeramente los Malacostraca (Figura 4).

Las pruebas entre la procedencia y Especie $\mathrm{y}$ Orden resultaron significativas, con $\chi_{(26)}^{2}=$ 40,943, $p=0,031$ (Figura 2), $\chi^{2}{ }_{(13)}=23,398, p=$ 0,037 , respectivamente. Por otro lado, la prueba estadística entre la procedencia y Clase no resultó significativa $\chi_{(4)}^{2}=6,867, p=0,143$ (Figura 3). Mientras las mariposas, las chinitas y las arañas pollitos fueron populares tanto en escuelas urbanas como en rurales, los escorpiones y las arañas no fueron tan preferidas en las escuelas rurales como lo fueron en las escuelas urbanas. Por lo tanto, al nivel de Orden, Scorpiones fue menos preferido en las escuelas rurales y al nivel de Clase Arachnida

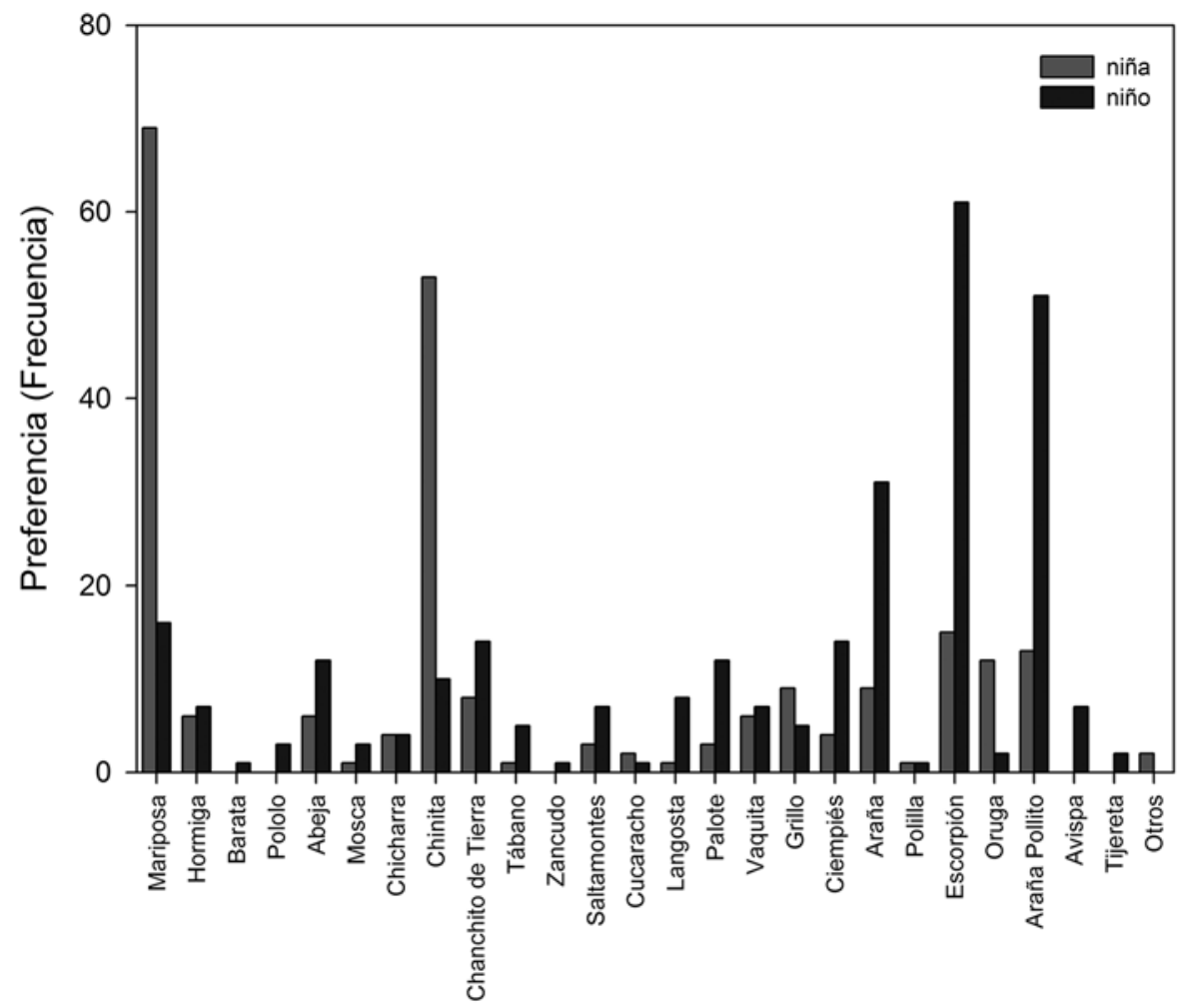

Organismo

Figura 2. Preferencia global para los diferentes tipos de artrópodos, separadas por sexo. 


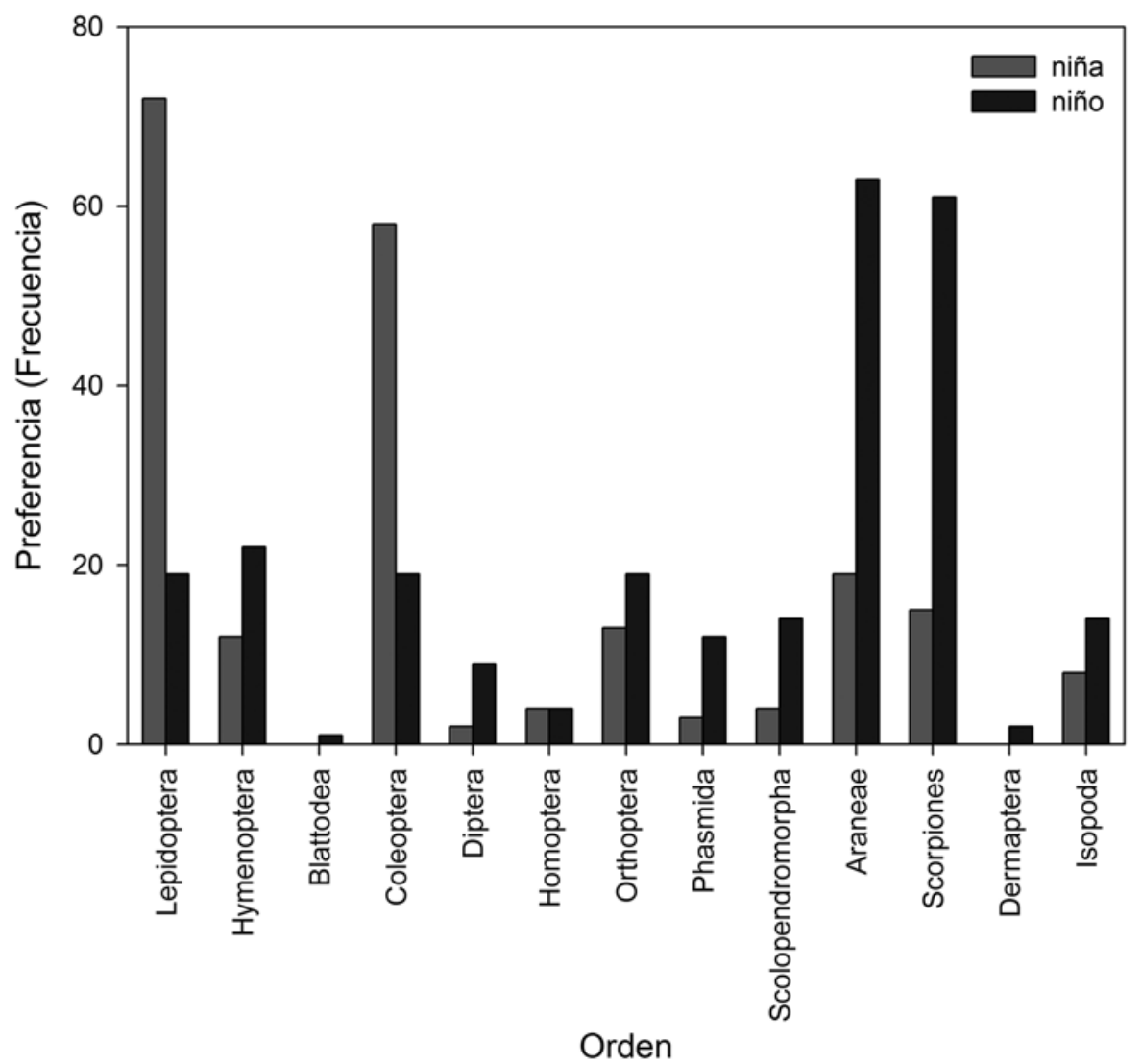

Figura 3. Preferencia global para los distintas Órdenes de artrópodos, separadas por sexo.

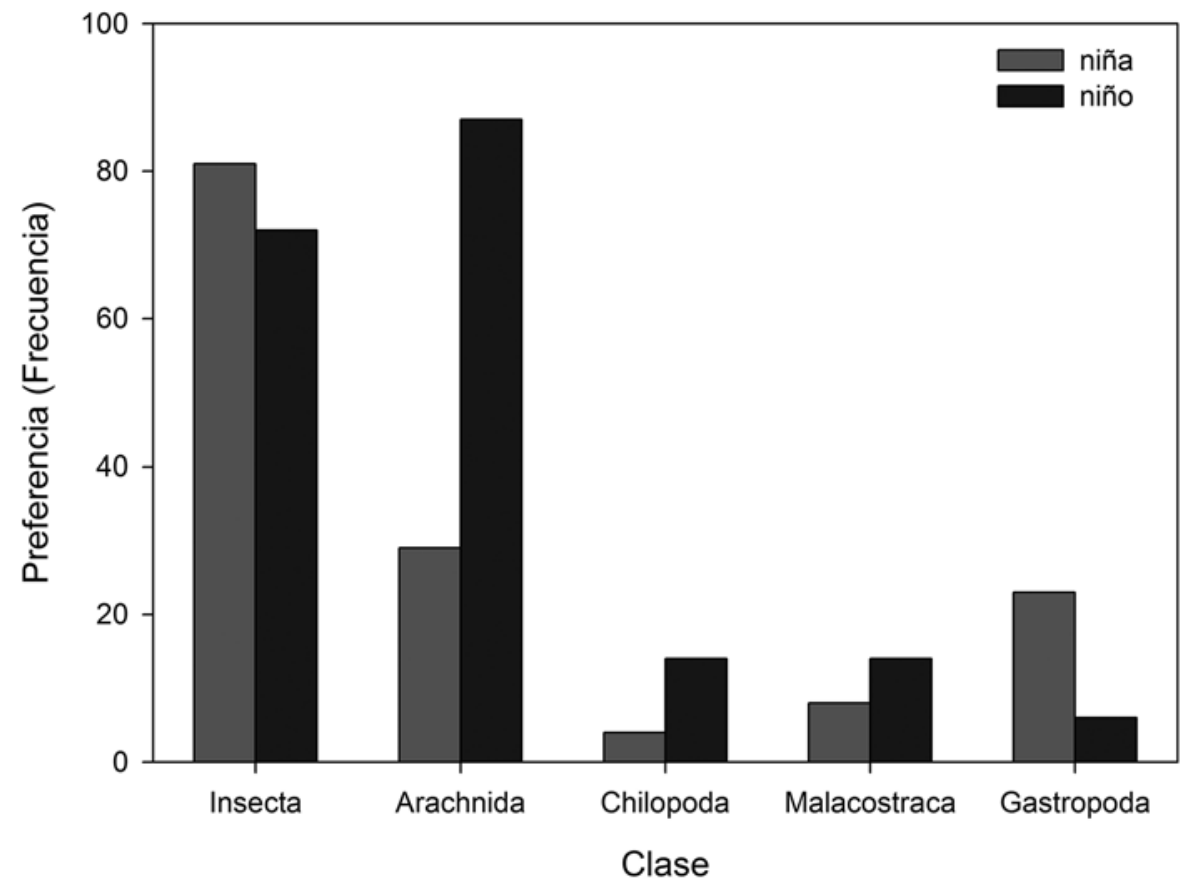

Figura 4. Preferencias separadas para las distintas Clases de artrópodos, separadas por sexo. 
fueron más preferidas, aunque no significativamente, en las escuelas urbanas.

\section{Conocimiento morfológico de los artrópodos epigeos en niños y niñas}

En el análisis de los dibujos de los alumnos de cursos inferiores a Quinto Año Básico $(X=1,42$, $\sigma=0,765)$ recibieron puntajes más bajos que los alumnos de Quinto Básico y superior $(X=1,60$, $\sigma=0,906)$, aunque los resultados del ANOVA no fueron significativos $\left(F_{(1,168)}=1,114, p=0,293\right)$. Esto muestra que los alumnos no presentan una mejoría significativa en los niveles de aprendizaje de las características morfológicas de los artrópodos después de haberles sido enseñadas (en Cuarto Año Básico), y en general, los alumnos no tienen un conocimiento morfológico aceptable sobre los artrópodos. Esto sugeriría que es preciso realizar más investigaciones para entender la baja representación de ciertos caracteres, especialmente los tagmas, en los dibujos de los participantes. Al analizar los dibujos removiendo la variable del tagma, tampoco se encuentran diferencias significativas entre niños bajo y sobre el Quinto Año Básico de Educación $\left(F_{(1,168)}=0,228, p=0,634\right)$. Esto muestra que aún sin la inclusión de la categoría tagmas, los alumnos mayores no dibujaron artrópodos más precisos que los alumnos menores.

\section{Discusión}

En el presente estudio las diferencias entre distintas edades no resultaron significativas, esto podría explicarse en base a que en el sistema educativo chileno, los niños tempranamente aprenden a diferenciar y a clasificar distintos tipos de organismos animales (MINEDUC, 2003a), aun cuando el énfasis está puesto en los animales vertebrados, más que en los grupos de animales invertebrados (MINEDUC, 2003a). En este sentido, el Mapa de Progreso del Aprendizaje de Estructura y Función de los Seres Vivos y el Plan y Programa de Curso de Cuarto Año Básico especifican que los niños y niñas deben reconocer que los animales y plantas pueden ser agrupados de acuerdo con criterios morfológicos de clasificación (i.e., mamíferos, aves, peces, etc.) (MINEDUC, 2003a, 2003b). Uno de los indicadores de logro de esta habilidad es que usando categorías convencionales puedan clasificar a animales vertebrados e invertebrados (insectos y arañas). Para estos últimos se reconocen como caracteres el número de patas, tagmas, presencia o ausencia de antenas, presencia o ausencia de alas y forma de desplazamiento (MINEDUC, 2003a). Esto sugiere que el tiempo de exposición al trabajo con tales categorías podría ser insuficiente y que las habilidades referidas a clasificar deben tener una mayor representación temporal.

Otro factor que resulta importante de considerar es el lugar en el cual los niños viven y asisten a la escuela (i.e., urbano, rural), ello puede relacionarse con la falta de experiencias con la naturaleza (Miller, 2005) o tiempos de exposición al entorno natural distintos (Tomazic, 2011). En esta investigación se sugiere que las personas que viven en lugares rurales -y por lo tanto posee más experiencia directa con la naturaleza- presenten percepciones ambientales diferentes y más amplias, lo que coincidiría con lo planteado por Strommen (1995), quien encontró diferencias entre niños y niñas de distintos lugares de procedencia en el nivel de conocimiento y en las concepciones de los bosques y de los animales que viven en ellos. Otras investigaciones demuestran que en los niños y niñas de zonas urbanas predomina el razonamiento homocentrista y de bienestar frente a la importancia de la flora y fauna (Howe et al., 1996).

\section{Preferencia taxonómica de los niños en relación con los artrópodos epigeos}

El hecho de que en este estudio se evidencie que existen preferencias de interés para algunos artrópodos, se corresponde con los antecedentes que muestran que los niños tienen preferencias para ciertas especies de artrópodos en particular, lo que se explicaría en función del género (Snaddon y Tuner, 2007; Tomkins y Tunnicliffe, 2007), ya que los niños suelen presentar preferencias por artrópodos venenosos, peligrosos o depredadores (Prokop et al., 2010). Este resultado es relevante, ya que se ha demostrado que tanto las preferencias como los conocimientos tienen consecuencias sobre la conservación biológica (Legare et al., 2009). Las preferencias y el interés que la población en general presenta por algunos artrópodos pueden verse sesgadas, ya que algunas especies de artrópodos pueden estar sobrerrepresentados en los medios de comunicación y en la literatura científica (Snaddon y Turner, 2007).

Es posible entonces, de acuerdo con lo analizado, que artrópodos más importantes en 
su papel biológico sean ignorados en iniciativas de conservación simplemente porque no son tan populares (Legare et al., 2009). En este sentido, es crucial el papel que juega la educación (tanto formal como informal) en entregar a los niños y niñas herramientas que le permitan mejorar sus conocimientos y actitudes hacia el entorno. El sistema educativo formal debe considerar los intereses, las preconcepciones y las preferencias que tienen los niños acerca de los animales en general, y de los insectos en particular, ya que no necesariamente preferirán a aquellos grupos para los cuales se dispone de mayor conocimiento (Tomkins y Tunnicliffe, 2007). Se ha demostrado que la educación puede utilizar a los insectos para incentivar el interés de los alumnos por el aprendizaje de las ciencias, incluyendo las ciencias ambientales (Matthews et al., 1997) y que las tendencias aprendidas durante la niñez son importantes para el interés futuro por las ciencias (Howe et al., 1996; Matthews et al., 1997).

\section{Diferencias entre las preferencias de los artrópodos epigeos, en relación con el género y edad de los niños y niñas}

Las diferencias significativas encontradas son concordantes con otras investigaciones que muestran que las diferencias en las preferencias a los artrópodos están ligadas al género y a la procedencia de los niños encuestados (Prokop et al., 2008). En nuestro caso, el factor edad no afectó significativamente los resultados, lo que es contrario a los antecedentes ya discutidos (Matthews et al., 1997; Shepardson, 2002) y puede explicarse por la baja habilidad de los estudiantes, independiente de su edad, de diferenciar entre los artrópodos como grupos separados a insectos de arácnidos. Una explicación posible a los resultados obtenidos en relación con sexo es que los niños y las niñas eligen artrópodos que siguen los estereotipos del sexo; ya que las mariposas y chinitas son artrópodos asociados culturalmente más al género femenino y las arañas y escorpiones al género masculino. En este sentido, estas diferencias en las preferencias pueden ser productos de la cultura en la que se encuentran inmersos y específicamente de los medios masivos de comunicación que transmiten y reproducen estos estereotipos (Ramadoss y Poyya-Moli, 2012). Considerando la influencia de la tecnología durante la niñez esto puede ser problemático, ya que los niños prestan más atención a ese mundo fabricado que a la realidad (Balmford et al., 2002).

\section{Conocimiento morfológico de los artrópodos epigeos en niños y niñas}

La investigación de Tunnicliffe y Reiss (1999) mostró que los niños de distintas edades utilizan rasgos anatómicos y físicos para categorizar animales y explicar las funciones y vidas de tales animales. También mostraron que la manera de categorizar de los niños es muy diferente de la categorización científica, porque los niños tienen muchas dificultades para entender. No sabemos lo que ocurre con otros grupos de organismos (e.g., plantas vasculares, animales vertebrados), que tienen una mayor representación en el currículum escolar de Educación General Básica (MINEDUC, 2003a, 2003b), pero advertimos que este modelo reproduce los sesgos de conocimiento de la biota nacional, en la cual el énfasis está puesto en los grupos más conocidos.

El escaso conocimiento morfológico sobre los artrópodos pueden dar cuenta de un nivel menor de comprensión, tanto de generación como de aplicación de criterios comunes para clasificar a estos organismos (MINEDUC, 2003a, 2003b). Por lo general los niños y niñas no tienen conocimiento morfológico de los artrópodos, y les resulta especialmente difícil diferenciar entre insectos y artrópodos no insectos (Shepardson, 2002); aunque los criterios necesarios para realizarlo se encuentran presentes en el currículum escolar (MINEDUC, 2003a). Esto sugiere que la educación formal no estaría interviniendo de una manera efectiva en establecer los contactos que vinculen a los niños y niñas con estas experiencias, ni el desarrollo de la habilidad esperada al usar como ejemplo de estudio a los artrópodos (Tomkins y Tunnicliffe, 2007). Esta sería una instancia particularmente importante como punto de intervención, ya que sabemos que las competencias sociales, emocionales y cognitivas que hayan desarrollado en sus primeros años de vida condicionarán el comportamiento por el resto de la vida (2021 Metas Educativas, 2010).

\section{Conclusiones}

La enseñanza de la ciencia en general y de los insectos en particular requieren experiencias interactivas y directas (Solervicens et al., 2007; 
Camus, 2009). Desafortunadamente la educación formal no estaría proveyendo a los niños de estas experiencias (Tomkins y Tunnicliffe, 2007; Camus, 2009). Pero hay otras fuentes para adquirir y/o aumentar esas experiencias y de hecho las investigaciones que existen sobre las percepciones y los conocimientos ambientales de los niños han mostrado que ellos dependen más de sus experiencias fuera de la escuela (Shepardson et al., 2007; Prokop et al., 2008, 2011a, 2011b). Por eso es importante considerar no solo la educación formal sino la educación informal, creando un perfil total e integrado de la educación de un niño. A modo de ejemplo, los niños que viven en lugares urbanos no van a tener tantas oportunidades de tener experiencias directas con la naturaleza, un hecho que los educadores deben tener en cuenta al desarrollar el currículo (Strommen, 1995; Howe et al., 1996), ya que modificaciones en los contenidos curriculares, enfatizando la perspectiva afectiva y emotiva, generan el ambiente adecuado para un proceso de alfabetización científica y de responsabilidad frente a la protección de los recursos disponibles.

El presente trabajo ha documentado algunos patrones interesantes e importantes sobre las relaciones entre los niños y niñas y los artrópodos. Pero también denota el escaso conocimiento que ellos tienen de estos taxa. Una manera de resolver parcialmente este problema es desarrollar más investigaciones y a escala escolar incorporar más experiencias interactivas que atiendan a las bases de la clasificación de los organismos en grandes grupos.
Por ahora proveemos de antecedentes referidos a los artrópodos; sin embargo, desconocemos el nivel de conocimiento y las preferencias que niños y niñas tienen de grupos de organismos que cuentan con una mayor representación en currículum, tales como plantas vasculares y animales vertebrados y si los esfuerzos de conservación coinciden con esas preferencias. En este trabajo se pone de manifiesto la necesidad de considerar de manera más explícita los factores afectivos en la educación formal, como también los aspectos actitudinales como elementos de aprendizaje y enseñanza de la educación científica. Además consideramos que se incorpore el rol que aspectos tales como los estereotipos, la falta de experiencia directa y el uso indiscriminado de la tecnología en la vida cotidiana ejercen sobre las preferencias y nivel de conocimiento de representantes, tanto de la fauna como de la flora local y nacional.

\section{Agradecimientos}

A los estudiantes y profesores de los establecimientos educacionales que participaron en la presente investigación. A los Programas EXPLORACONICYT, Región de Coquimbo y ECBI-Región de Coquimbo, Chile. Al Departamento de Biología y a la Facultad de Ciencias, Universidad de La Serena. Al Convenio Universidad de La Serena con C.V. Starr-Middlebury College-School in Latin AmericaChile (F.L.C.). Proyecto financiado por Proyecto DIULS-PR13121 de la Dirección de Investigación, Universidad de La Serena, La Serena (J.P.A).

\section{Literatura Citada}

\section{Metas Educativas}

2010. La educación que queremos para la generación de los bicentenarios, documento final. Organización de Estados Iberoamericanos para la Educación, la Ciencia y la Cultura, Barcelona, España. 151 pp.

Balmford, A.; Clegg, L.; Coulson, T.; Taylor, J.

2002. Why conservationists should heed Pokémon. Science, 295: 2367.

Bixler, R.; Floyd, M.; Hammitt, W.

2002. Environmental socialization: Quantitative tests of the childhood play hypothesis. Environment and Behavior, 34: 795-818.

Camus, P.A.

2009. Educación científica y evolutiva en Chile: problemas funcionales y conflictos entre enseñar y aprender. Gayana, 73: 19-31.
Cepeda-Pizarro, J.; Pizarro-Araya, J.; Vásquez, H.

2005. Composición y abundancia de artrópodos epigeos del Parque Nacional Llanos de Challe: impactos del ENOS de 1997 y efectos del hábitat pedológico. Revista Chilena de Historia Natural, 78: 635-650.

Gutiérrez, J.R.

2008. El desierto florido en la Región de Atacama. En: Squeo, F.A.; Arancio, G.; Gutiérrez, J.R. (eds.). Libro Rojo de la Flora Nativa y de los Sitios Prioritarios para su conservación: Región de Atacama. Ediciones Universidad de La Serena, La Serena, Chile. Pp. 285-291.

Howe, D.; Kahn, P.; Friedman, B.

1996. Along the Río Negro: Brazilian children's environmental views and values. Developmental Psychology, 32: 979-987. 
Legare, C.; Wellman, H.; Gelman, S.

2009. Evidence for an explanation advantage in naïve biological reasoning. Cognitive Psychology, 58: 177-194.

Matthews, R.; Flage L.; Matthews, J.

1997. Insects as teaching tools in primary and secondary education. Annual Review of Entomology, 42: 269-289.

Miller, J.

2005. Biodiversity conservation and the extinction of experience. Trends in Ecology and Evolution, 20: 430-434.

MINEDUC

2003a. Programa de Estudio Cuarto Año Básico. Comprensión del Medio Natural, Social y Cultural. Ministerio de Educación, Santiago, Chile. 66 pp.

MINEDUC

2003b. Programa de Estudio Tercer Año Básico. Comprensión del Medio Natural, Social y Cultural. Ministerio de Educación, Santiago, Chile. 62 pp.

Pimm, S.; Russell, G.; Gittleman, J.; Brooks, T. 1995. The future of biodiversity. Science, 269: 347-350.

Prokop, P.; Tunnicliffe, S.D.

2010. Effects of having pets at home on children's attitudes toward popular and unpopular animals. Anthrozoos, 23: 21-35.

Prokop, P.; Prokop, M.; Tunnicliffe, S.D.

2008. Effects of keeping animals as pets on children's concepts of vertebrates and invertebrates. International Journal of Science Education, 30: 431-449.

Prokop, P.; Usak, M.; Erdogan, M.; Fancovicova, J.; Bahar, M. 2011a. Slovakian and Turkish students' fear, disgust and perceived danger of invertebrates. Hacettepe Universitesi Egitim Fakultesi Dergisi-Hacettepe University Journal of Education, 40: 344-352.

Prokop, P.; Usak, M.; Erdogan, M.

2011b. Good predators in bad stories: cross-cultural comparison of children's attitudes towards wolves. Journal of Baltic Science Education, 10: 229-242.

Ramadoss, A.; Poyya-Moli, G.

2012. Field Based Learning about Butterfly Diversity in School Garden-A Case Study From Puducherry, India.
International Electronic Journal of Environmental Education, 2: $149-154$.

Shepardson, D.

1997. Of butterflies and beetles: First graders' ways of seeing and talking about insect life cycles. Journal of Research in Science Training, 34: 873-889.

Shepardson, D.

2002. Bugs, butterflies, and spiders: children's understandings about insects. International Journal of Science Education, 24: 627-643.

Shepardson, D.; Wee, B.; Priddy, M.; Harbor, J.

2007. Students' mental models of the environment. Journal of Research in Science Teaching, 44: 327-348.

Snaddon, J.L.; Turner, E.

2007. A child's eye view of the insect world: perceptions of insect diversity. Environmental Conservation, 34: 33-35.

Solervicens, J.; Estrada, P.; Chiappa, E.; Alviña, A.

2007. Cómo trabajar con insectos en la sala de clases. Fondo Editorial Universidad Metropolitana de Ciencias de la Educación. Santiago, Chile. 106 pp.

Steel, R.G.D.; Torrie, J.H.

1989. Bioestadística. Principios y Procedimientos. Editorial Graf América. México, 622 pp.

Strommen, E.

1995. Lions and tigers and bears, oh my! Children's conceptions of forests and their inhabitants. Journal of Research in Science Teaching, 32: 683-698.

Thomas, D.; Decady, Y.

2000. Analyzing categorical data with multiple responses per subject. SCC Annual Meeting, Proceedings of the Survey Methods Section, 121-130.

Tomkins, S.; Tunnicliffe, S.

2007. Nature tables: stimulating children's interest in natural objects. Journal of Biological Education, 41: 150-155.

Tunnicliffe, S.; Reiss, M.

1999. Building a model of the environment: how do children see animals? Journal of Biological Education, 33: 142-148. 
